$15^{\text {th }}$ International Conference on

AEROSPACE SCIENCES \& AVIATION TECHNOLOGY,

$\boldsymbol{A S A T}$ - 15 - May 28 - 30, 2013, Email: asat@mtc.edu.eg,

Military Technical College, Kobry Elkobbah, Cairo, Egypt,

Tel: +(202) 24025292 -24036138, Fax: +(202) 22621908

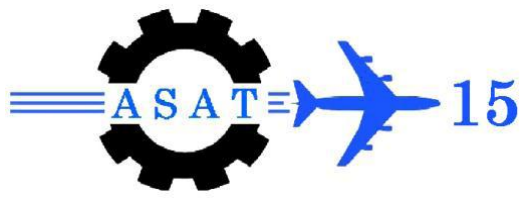

\title{
FE and Experimental Modeling of On-shaft Vibration Measurement
}

\author{
M. E. Elnady ${ }^{*}$, A. Abdelbary ${ }^{*}$, J. K. Sinha ${ }^{\dagger}$, S. O. Oyadiji ${ }^{\dagger}$
}

\begin{abstract}
Vibration-based condition monitoring is a well-accepted tool for diagnosing rotating machines faults. Measuring vibration on the bearings uses multiple sensors and needs skilled personnel for data collection, processing and interpretation. An analytical model for the alternate method of On-Shaft Vibration Measurement is presented. The model is validated through FE and experimental analysis. The results show that the model efficiently represents the measurement process.
\end{abstract}

Keywords: Vibration-based condition monitoring, on-shaft vibration measurement, MEMS accelerometer, wireless sensor nodes.

\section{Introduction}

\subsection{Vibration-Based Condition Monitoring}

Rotating machines form the backbone of almost all mechanical systems. Usually, wherever motion is required, a rotating machine would exist running smoothly and efficiently to provide the motive force.

For Vibration-based Condition Monitoring (VCM), the vibration sensor is placed on the bearing pedestals and the shaft vibration is indirectly measured. In some cases, it is directly measured from the shaft itself. Mitchell [1] discussed measurement arrangements for commonly monitored machines (centrifugal pumps, axial compressors, fans, gas generators, etc.).

These setups lay within On-Bearing Vibration Measurement (OBVM) which is a reliable condition monitoring tool, however some limitations are observed:

a) Highly experienced personnel are required to collect and analyze the data.

b) On-bearing sensors are generally affected by structural noise propagating through the machine hull.

c) A considerable space is needed for the system components and cabling.

d) The system capital and maintenance costs are relatively high.

e) One of the ways to enhance the Faults Diagnosis (FD) is to increase the number of sensors [2]. However, this necessitates exhaustive signal processing work.

f) More research is required to identify robust and unique fault features especially when multiple faults coexist.

For these reasons, vibration analysts have been dreaming of a method to capture the shaft vibration directly without being affected by bearing and structure imperfections, journal bearing damping, structural noise, etc. Also, reduction of the installed sensors merely minimizes the system maintenance cost and the signal processing.

\footnotetext{
*Egyptian Armed Forces, Egypt.

${ }^{\dagger}$ University of Manchester, UK.
} 


\subsection{On-Shaft Vibration Measurement (OSVM)}

Conceptually, OSVM seeks acquiring the vibration at its source. That is the rotor (for rotorrelated faults) where its amplitude has its maximum value. Accordingly, faults symptoms are expected to be much more detectable. A sensor fixed to the shaft rotates by nature; see Figure 1(a). This means that a single sensor collects data along the two axes forming its plane of rotation. Theoretically, a single on-shaft sensor may replace two or four sensors per bearing.

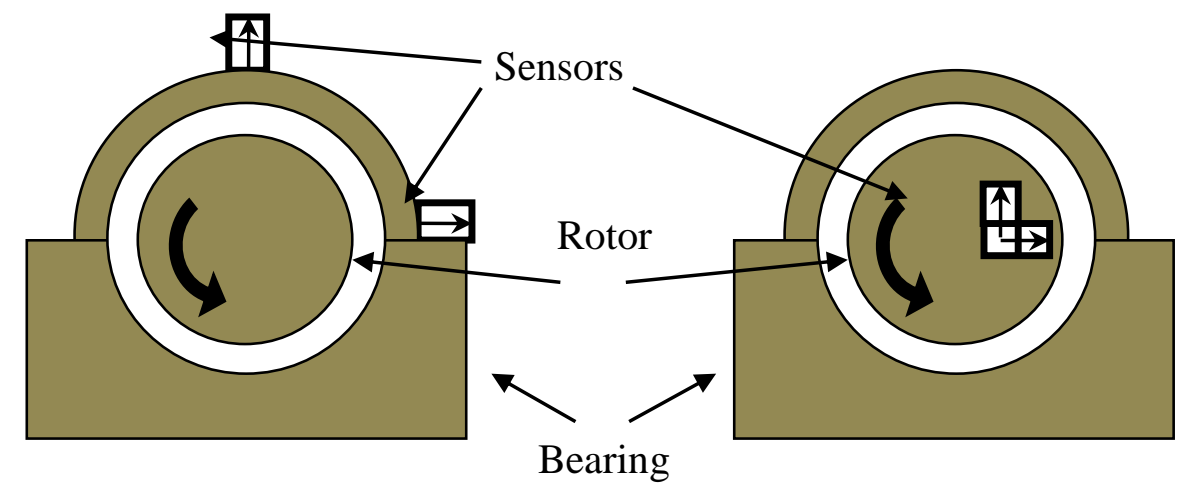
(a)
OBVM
(b)
OSVM

\section{Figure 1: Sensor positions in vibration-based condition monitoring: (a) OBVM and (b) OSVM.}

OSVM started with torsional vibration telemetry measurement systems. One of the early trials was introduced by Lees et al. [3]. They apply a strain gauge with a telemetry unit that transmits its output to a stationary receiver. The receiver antenna is usually a circular ring surrounding the rotor at the transmitter position. These systems are well accepted in industry $[4,5]$ and gave better insight into the shaft vibration. However, the cumbersome setup hindered their permanent installation on rotors. More importantly, many faults express themselves in the lateral vibration rather than the torsional vibration one. The latter is known to be less informative than the former from the faults diagnosis point of view. Nevertheless, torsional vibration gives good gear faults diagnosis [6].

The continuing advancement in sensor and data acquisition hardware technologies enabled researchers to attach different types of vibration sensors, including accelerometers, along with miniaturized Wireless Sensor Nodes (WSN) on the rotor. The lateral vibration is now measurable which promises a wide range of faults to be detected.

A major point to be considered for OSVM is the rotary motion of the sensor. For OBVM, both accelerometers are aligned orthogonal to each other and keep their directions with time. On the contrary, an accelerometer on the shaft will keep alternating between two orthogonal planes. Instead of having two independent lateral vibration channels, we would get two largely similar ones each of which contains coupled (horizontal and vertical) vibration responses. This leads us to an inevitable question: "To what extent can we use OSVM in rotor dynamics analysis and faults diagnosis work?" In order to find an answer for this question some researchers investigated OSVM.

Arebi et al. [7] used an on-shaft accelerometer and an encoder to measure the tangential acceleration and the instantaneous angular speed respectively. They monitored the changes in the harmonics amplitudes with speed and misalignment severity and used it successfully to detect rotor misalignment. However, they noticed incomplete conformance between the harmonics from the accelerometer and those from the encoder. 
Another work introduced by Baghli et al. [8] in order to consider the rotational speed variation is measured by the tangential on-shaft sensor. The variation was correlated with the net torque (difference between electromagnetic and load torques), hence can be used in the diagnosis of motor faults. Additionally, they used the radial sensor output to estimate speed as it is affected by the centrifugal acceleration. The estimated speed matched with that measured using an encoder limited by the measurement range of the radial sensor to $350 \mathrm{rpm}$. It also showed higher fluctuations since it is proportional to the squared rotational speed. To eliminate the gravity effect, they used two opposing micro-electro- mechanical systems (MEMS) accelerometers soldered to a Printed Circuit Board (PCB) surrounding the rotor. At any instant of time, the gravity field affects both accelerometers with the same magnitude but with different signs. The direct addition of their outputs produces a gravity free signal. This arrangement reduces the applicability of OSVM especially for large diameter rotors.

Thompson [9] used a MEMS accelerometer bonded to a fan blade to monitor the centrifugal acceleration imposed on it. He used only a radial accelerometer and therefore was limited to a high range and low sensitivity accelerometer type.

As well as accelerometer-based OSVM systems, Sloetjes et al. [10] bonded a strain gauge to a rotor and used the measured vibration amplitude as an input to a vibration reduction system. Although this may be the only article that discussed the critical speed crossing while using OSVM, they did not analyze the vibration spectrum and consequently very important observations was missed.

Reviewing the research on OSVM the following drawbacks are identified:

a) Most studies reported limited observations and did not consider how the measurement process works.

b) OSVM has not been applied in rotor dynamics analysis of lateral vibration.

c) Most of test rigs used have high critical speeds. This prevented important observations being made.

In this study, the above mentioned drawbacks are considered, disadvantages are turned into advantages and a better understanding of OSVM is established.

\section{Test Rig and FE Model}

\subsection{Test Rig}

The test rig (Figure 2) is a modified version of the rig used earlier [11, 12] to investigate OSVM during constant speed [11] and during run-up [12]. In Elnady et al. [11], the rotor used to be short and mounted on rigid supports (with a critical speed of $68 \mathrm{~Hz}$ ). In that configuration, the signal produced by the on-shaft sensor was only due to the shaft rotation with negligible rotor vibration. This proved the feasibility of the proposed OSVM system and also revealed the gravity effect within the sensor output.

In Elnady et al. [12], the rotor length has been increased to attain lower critical speeds (34 and $35 \mathrm{~Hz}$ in horizontal and vertical directions respectively). Upon conducting run-ups, the response amplifications upon crossing integer fractions of the critical speeds where very clear. However, response modulation was observed and reported as a major finding. 


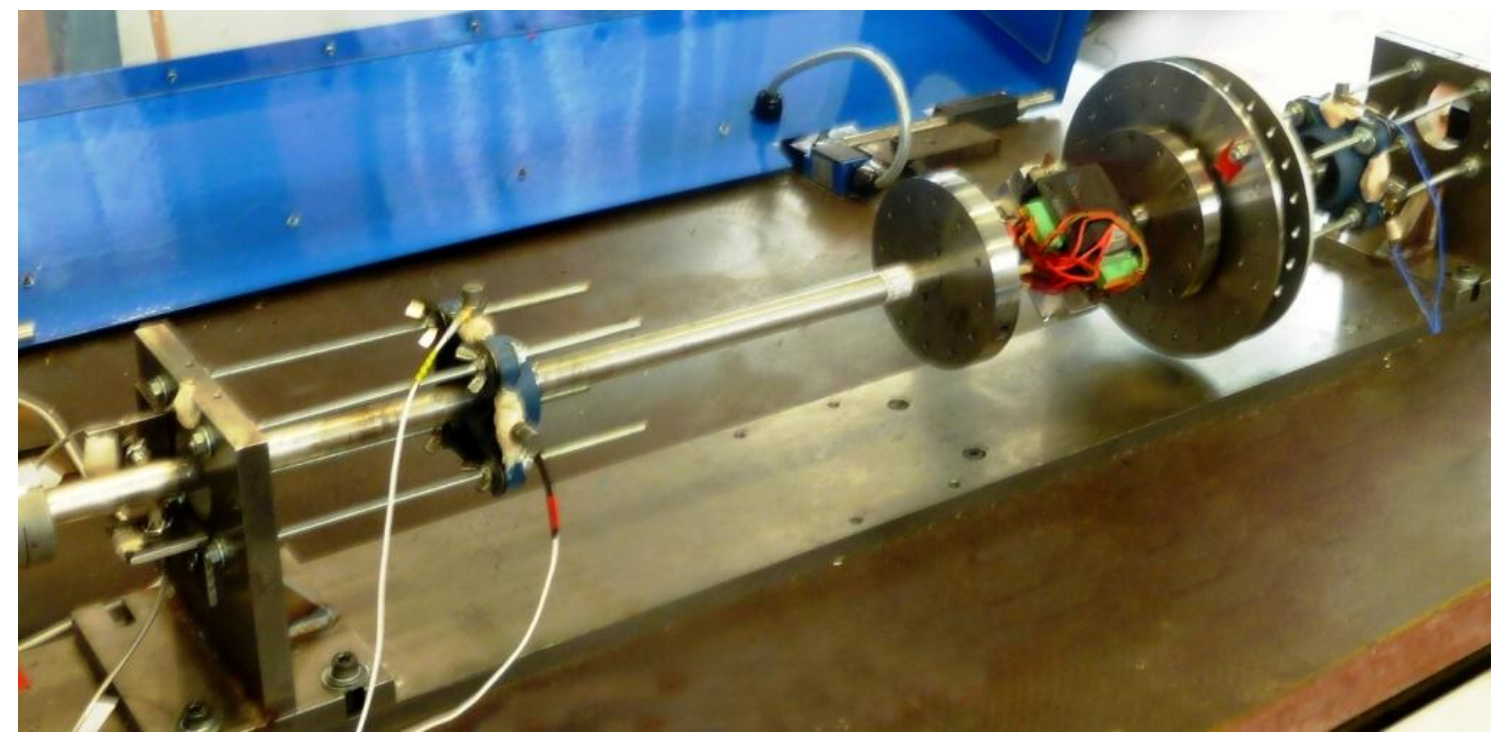

Figure 2: Test rig.

In the current work, it is required to get critical speeds less than the maximum speed of the rotor $(25 \mathrm{~Hz})$. This is done by two measures; increased pedestal flexibility and increased rotor mass. Flexible supports are used to mount the bearing on the pedestals [13]. Additional discs are used to increase the rotor mass. These two modifications yielded two critical speeds of 11.8 and $13.66 \mathrm{~Hz}$ in the vertical and horizontal directions respectively.

\subsection{FE Model}

The purpose of this FE modeling is to build a mathematical model for the test rig that can be used later in discussing the OSVM process through comparison between simulated and experimental responses. The procedure starts by modeling the rig components individually [13]. Figure 3 depicts a graphical representation of the FE model. The associated mass and stiffness matrices are then computed and assembled before being substituted in the overall equation of motion.

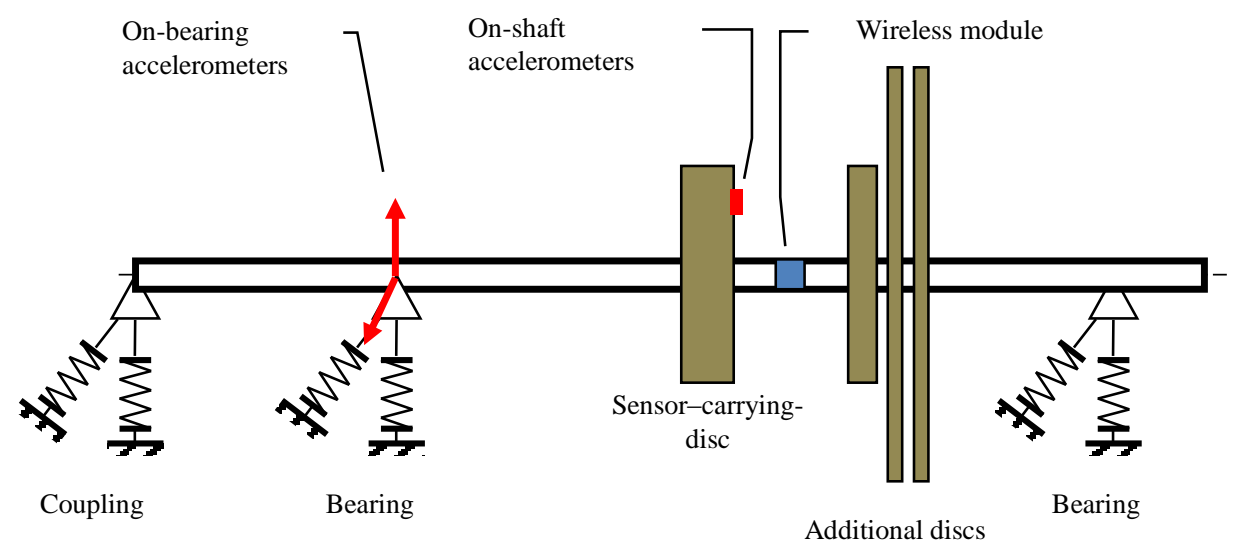

Figure 3: Graphical representation for the FE model

Throughout the modeling process, all the materials are assumed to be isotropic. The shaft has a slender geometry, therefore, its rotary inertia and deformation due to shear can be neglected. The Euler-Bernoulli beam theory is adopted to build its stiffness and mass matrices. Discs are considered rigid and have no effect on the shaft stiffness. The shaft is discretized into 50 elements and each component occupies one node. The wireless module is modeled as a point mass. 
To describe the motion of each node in the model in terms of its spatial coordinates, it is necessary to define a generalized coordinates vector that includes sufficient degrees of freedom. Each node is free to translate and rotate along two axes ( $\mathrm{x}$ and $\mathrm{y}$ ), hence a four element vector is sufficient.

$$
\mathrm{q}=\left[\begin{array}{c}
\mathrm{u} \\
\mathrm{v} \\
\theta \\
\psi
\end{array}\right]
$$

where $\mathrm{u}$ and $\theta$ are the translation and rotation coordinates for the $\mathrm{x}$-axis respectively and $\mathrm{v}$ and $\psi$ are the translation and rotation coordinates for the y-axis respectively.

According to the coordinate system, a two-node shaft element can be represented as shown in Figure 4, where $\omega$ is the shaft angular speed and the suffix number for $\mathrm{u}, \mathrm{v}, \theta$ and $\psi$ denotes the node number.

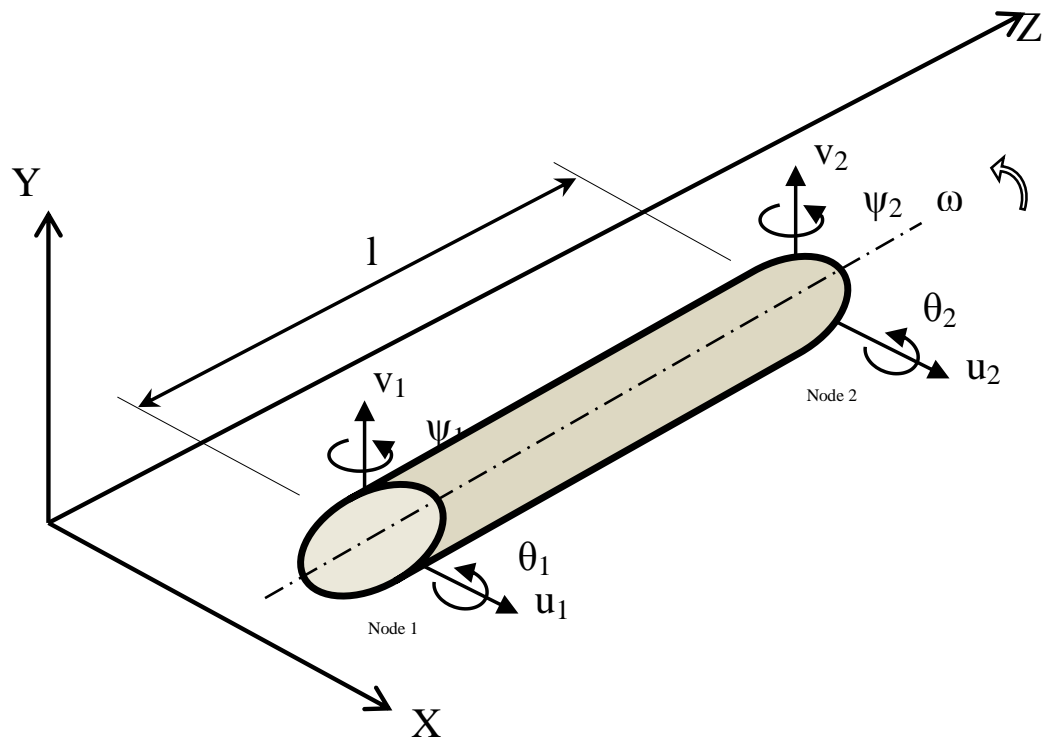

Figure 4: Geometry of the used shaft element.

For each element, the mass $\mathbf{M}_{\mathrm{e}}$ and stiffness $\mathbf{K}_{\mathrm{e}}$ matrices are calculated according to the Euler-Bernoulli beam theory as:

$$
\mathrm{M}_{\mathrm{e}}=\frac{\rho_{\mathrm{e}} \mathrm{A}_{\mathrm{e}} l_{\mathrm{e}}}{420}\left[\begin{array}{cccccccc}
156 & 0 & 0 & 22 \mathrm{le} & 54 & 0 & 0 & -13 \mathrm{le}^{\mathrm{e}} \\
0 & 156 & -22 \mathrm{l}_{\mathrm{e}} & 0 & 0 & 54 & 13 \mathrm{l}_{\mathrm{e}} & 0 \\
0 & -22 \mathrm{l}_{\mathrm{e}} & 4 \mathrm{l}_{\mathrm{e}}^{2} & 0 & 0 & -13 \mathrm{l}_{\mathrm{e}} & -3 \mathrm{l}_{\mathrm{e}}^{2} & 0 \\
22 l_{\mathrm{e}} & 0 & 0 & 4 \mathrm{l}_{\mathrm{e}}^{2} & 13 \mathrm{l}_{\mathrm{e}} & 0 & 0 & -3 \mathrm{l}_{\mathrm{e}}^{2} \\
54 & 0 & 0 & 13 \mathrm{l}_{\mathrm{e}} & 156 & 0 & 0 & -22 \mathrm{l}_{\mathrm{e}} \\
0 & 54 & -13 \mathrm{l}_{\mathrm{e}} & 0 & 0 & 156 & 22 \mathrm{l}_{\mathrm{e}} & 0 \\
0 & 13 \mathrm{l}_{\mathrm{e}} & -3 \mathrm{l}_{\mathrm{e}}^{2} & 0 & 0 & 22 \mathrm{l}_{\mathrm{e}} & 4 \mathrm{l}_{\mathrm{e}}^{2} & 0 \\
-13 l_{\mathrm{e}} & 0 & 0 & -3 \mathrm{l}_{\mathrm{e}}^{2} & -22 \mathrm{l}_{\mathrm{e}} & 0 & 0 & 4 \mathrm{l}_{\mathrm{e}}^{2}
\end{array}\right](2)
$$




$$
\mathrm{K}_{\mathrm{e}}=\frac{\mathrm{E}_{\mathrm{e}} \mathrm{I}_{\mathrm{e}}}{l_{\mathrm{e}}^{3}}\left[\begin{array}{cccccccc}
12 & 0 & 0 & 6 l_{\mathrm{e}} & -12 & 0 & 0 & 6 l \mathrm{e} \\
0 & 12 & -6 l_{\mathrm{e}} & 0 & 0 & -12 & -6 l_{\mathrm{e}} & 0 \\
0 & -6 l_{\mathrm{e}} & 4 l_{\mathrm{e}}^{2} & 0 & 0 & 6 l_{\mathrm{e}} & 2 l_{\mathrm{e}}^{2} & 0 \\
6 l_{\mathrm{e}} & 0 & 0 & 4 l_{\mathrm{e}}^{2} & -6 l_{\mathrm{e}} & 0 & 0 & 2 l_{\mathrm{e}}^{2} \\
-12 & 0 & 0 & -6 l_{\mathrm{e}} & 12 & 0 & 0 & -6 l_{\mathrm{e}} \\
0 & -12 & 6 l_{\mathrm{e}} & 0 & 0 & 12 & 6 l_{\mathrm{e}} & 0 \\
0 & -6 l_{\mathrm{e}} & 2 l_{\mathrm{e}}^{2} & 0 & 0 & 6 l_{\mathrm{e}} & 4 l_{\mathrm{e}}^{2} & 0 \\
6 l_{\mathrm{e}} & 0 & 0 & 2 l_{\mathrm{e}}^{2} & -6 l_{\mathrm{e}} & 0 & 0 & 4 l_{\mathrm{e}}^{2}
\end{array}\right]
$$

where

$\rho_{\mathrm{e}} \quad$ density

$\mathrm{A}_{\mathrm{e}} \quad$ cross section area

$l_{\mathrm{e}} \quad$ length

$\mathrm{E}_{\mathrm{e}} \quad$ Young's modulus of Elasticity

$\mathrm{I}_{\mathrm{e}} \quad$ second moment of area

A disc occupies only one node, consequently a $4 * 4$ matrix $\mathbf{M}_{\mathbf{d}}$ is sufficient to describe its mass and inertia effects.

$$
\mathbf{M}_{\mathbf{d}}=\left[\begin{array}{cccc}
\mathrm{m}_{\mathrm{d}} & 0 & 0 & 0 \\
0 & \mathrm{~m}_{\mathrm{d}} & 0 & 0 \\
0 & 0 & \mathrm{I}_{\mathrm{d}} & 0 \\
0 & 0 & 0 & \mathrm{I}_{\mathrm{d}}
\end{array}\right]
$$

where

$\mathrm{m}_{\mathrm{d}}, \mathrm{I}_{\mathrm{d}}$ disc mass and diametral moments of inertia

The disc has no contribution to the overall stiffness matrix, however its inertia adds some effect to the gyroscopic matrix $\mathbf{G}_{\mathbf{d}}$

$$
\mathbf{G}_{\mathbf{d}}=\left[\begin{array}{cccc}
0 & 0 & 0 & 0 \\
0 & 0 & 0 & 0 \\
0 & 0 & 0 & \mathrm{I}_{\mathrm{p}} \\
0 & 0 & -\mathrm{I}_{\mathrm{p}} & 0
\end{array}\right]
$$

where

$\mathrm{I}_{\mathrm{p}} \quad$ disc polar moments of inertia

Obviously, the point mass has no moment of inertia, its mass matrix is:

$$
\mathbf{M}_{\mathbf{p}}=\left[\begin{array}{cc}
\mathrm{m}_{\mathrm{p}} & 0 \\
0 & \mathrm{~m}_{\mathrm{p}}
\end{array}\right]
$$

where

$\mathrm{m}_{\mathrm{p}} \quad$ point mass's mass

The bearing units allow the inner race to swivel; hence the angular stiffness terms are neglected. The cross stiffness terms are also neglected. The bearing stiffness matrix $\mathbf{K}_{\mathrm{b}}$ is constructed as follows:

$$
\mathbf{K}_{\mathbf{b}}=\left[\begin{array}{cc}
\mathrm{k}_{\mathrm{uu}} & 0 \\
0 & \mathrm{k}_{\mathrm{vv}}
\end{array}\right]
$$

where

$\mathrm{k}_{\mathrm{uu}}$, and $\mathrm{k}_{\mathrm{vv}} \quad$ translational stiffnesses in $\mathrm{x}$ and $\mathrm{y}$ directions respectively 
The bearing damping matrix $\mathbf{C}_{\mathrm{b}}$ is constructed as follows:

$$
\mathbf{C}_{\mathbf{b}}=\left[\begin{array}{cc}
\mathrm{c}_{\mathrm{uu}} & 0 \\
0 & \mathrm{c}_{\mathrm{vv}}
\end{array}\right]
$$

where

$\mathrm{c}_{\mathrm{uu}}$, and $\mathrm{c}_{\mathrm{vv}}$ damping in $\mathrm{x}$ and $\mathrm{y}$ directions respectively

For the coupling, the angular stiffness is considered. The coupling stiffness matrix $\mathbf{K}_{\mathrm{c}}$ is constructed as follows:

$$
\mathbf{K}_{\mathbf{c}}=\left[\begin{array}{cccc}
\mathrm{k}_{\mathrm{uu}} & 0 & 0 & 0 \\
0 & \mathrm{k}_{\mathrm{vv}} & 0 & 0 \\
0 & 0 & \mathrm{k}_{\theta \theta} & 0 \\
0 & 0 & 0 & \mathrm{k}_{\psi \psi}
\end{array}\right]
$$

where

$\mathrm{k}_{\mathrm{uu}}$, and $\mathrm{k}_{\mathrm{vv}} \quad$ translational stiffnesses in $\mathrm{x}$ and $\mathrm{y}$ directions respectively

$\mathrm{k}_{\theta \theta}$, and $\mathrm{k}_{\psi \psi} \quad$ rotational stiffnesses in $\mathrm{x}$ and $\mathrm{y}$ directions respectively

The overall model matrices can be built by assembling the individual components ones. The shaft is composed of 51 nodes, which results in 204 dofs (degrees of freedom). Hence, a shaft matrix would be of size $204 * 204$ elements. The rest of the rig elements are added to their respective nodes according to their relative positions on the rotor. The model will be adapted to match the rig in configurations $\mathrm{A}$ and $\mathrm{B}$ only.

The overall equation of motion can be written as [14]:

$$
\mathbf{M} \ddot{\mathbf{q}}(\mathrm{t})+(\mathbf{C}+\omega \mathbf{G}) \dot{\mathbf{q}}(\mathrm{t})+\mathbf{K} \mathbf{q}(\mathrm{t})=\mathbf{F}(\mathrm{t})
$$

where

M mass matrix

C, G damping and gyroscopic matrices

K stiffness matrix

$\mathbf{F}(\mathrm{t})$ force vector

The bearing and coupling damping and stiffness terms are estimated through modal testing of each component separately. The equation of motion is solved by a MATLAB code [14] which transforms it into a set of ordinary differential equations and solves them using the MATLAB function "ODE45".

\section{Method}

In this section, an analytical model for the OSVM process is derived. A plane is assumed to gather the sensor $(\mathrm{S})$, the disc center of gravity (CG), and the disc geometric center $(\mathrm{C})$.

Figure 5 represents this plane with the applicable accelerations and reference planes.

The shaft geometry center (C) rotates around center of rotation $(\mathrm{O})$ and the sensor is positioned at point $\mathrm{S}$. The gravity field $(\mathrm{G})$ acts downward. The shaft whirls in an inertial frame $(\mathrm{X}, \mathrm{Y})$. A rotating frame $(\mathrm{R}, \mathrm{T})$ shares the same origin with the inertial one and is aligned along the line connecting the shaft center to the sensor. 


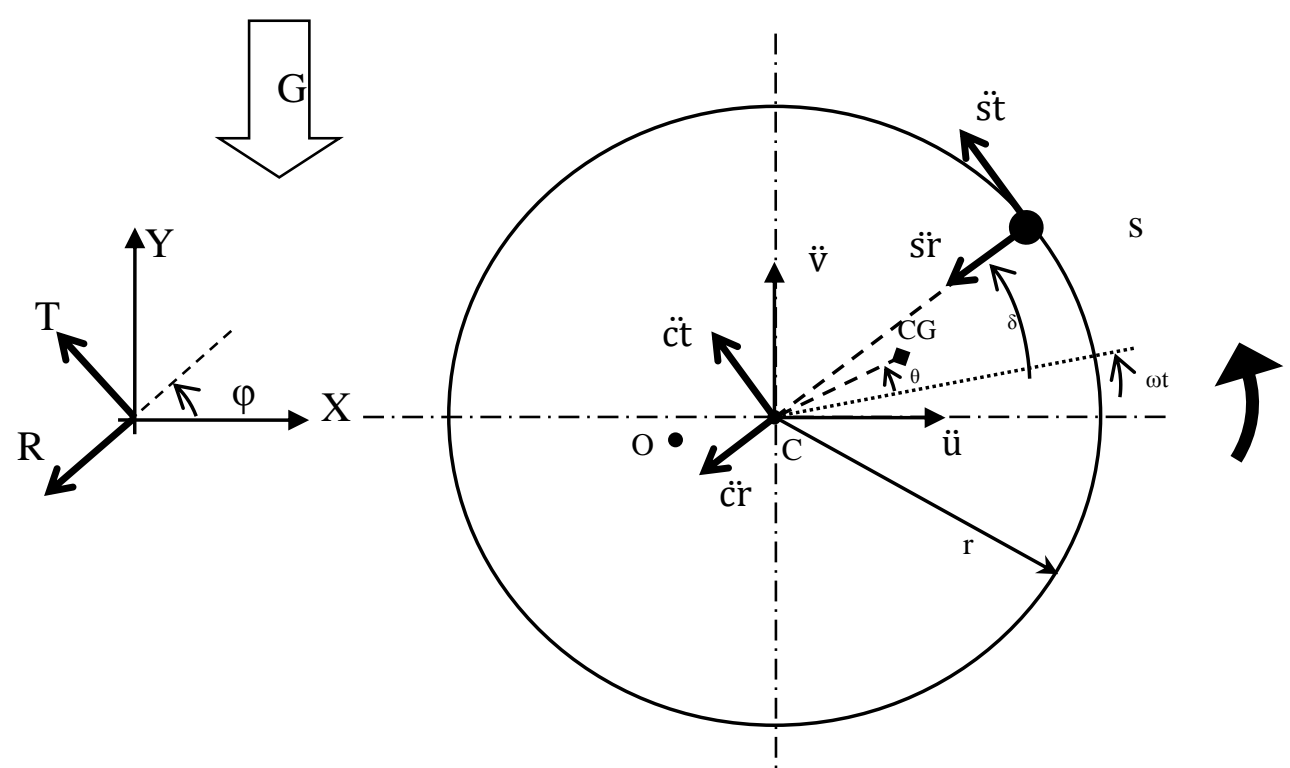

Figure 5: OSVM model geometry

where

CG shaft gravity center

$\mathrm{r} \quad$ sensor radial distance from the shaft center

$\Theta \quad$ unbalance phase

$\delta \quad$ sensor setting angle

$\ddot{\mathrm{s}}_{\mathrm{r}}, \ddot{\mathrm{s}}_{\mathrm{t}} \quad$ radial and tangential accelerations measured at the sensor position in the rotating frame (including gravity)

$\ddot{\mathrm{c}}_{\mathrm{r}}, \ddot{\mathrm{c}}_{\mathrm{t}} \quad$ radial and tangential accelerations at the shaft geometric center in the rotating frame $\ddot{u}, \ddot{v} \quad$ horizontal and vertical accelerations at shaft geometric center in the inertial frame

The objective is to predict the acceleration response at the sensor position in the rotating frame $(\mathrm{R}, \mathrm{T})$ given the acceleration response at the shaft center in the inertial frame $(\mathrm{X}, \mathrm{Y})$. We start from the displacement response at the shaft center in the inertial frame (X, Y) since it is the FE model output. This is done over four steps:

1. Calculating the velocity and acceleration responses at the shaft center in the inertial frame.

2. Transforming the response to the rotating frame using the axes rotation matrix.

3. Moving the calculation point to the sensor location using the principle of relative motion.

4. Adding the gravity effect.

These steps combine to form the following equation:

$$
\left[\begin{array}{c}
\ddot{\mathrm{s}}_{\mathrm{r}} \\
\ddot{\mathrm{s}}_{\mathrm{t}}
\end{array}\right]=\left[\begin{array}{cc}
-\cos \varphi & -\sin \varphi \\
-\sin \varphi & \cos \varphi
\end{array}\right] \cdot\left[\begin{array}{c}
\mathrm{U} \cos (\omega \mathrm{t}+\theta) \\
\mathrm{V} \sin (\omega \mathrm{t}+\theta)
\end{array}\right]+\left[\begin{array}{c}
-\omega^{2} \mathrm{r} \\
\dot{\omega} \mathrm{r}
\end{array}\right]+\mathrm{g}\left[\begin{array}{c}
-\sin (\varphi) \\
\cos (\varphi)
\end{array}\right]
$$

where $\mathrm{U}$ and $\mathrm{V}$ are acceleration response amplitudes at shaft center in $\mathrm{X}$ and $\mathrm{Y}$ directions respectively. 


\section{Experiments}

A run up is conducted up to a speed of $950 \mathrm{rpm}$ along 2 minutes; Figure 6 shows the time waveforms for OBVM and OSVM.

OBVM is measured in the horizontal, On-Bearing Horizontal $(\mathrm{OBH})$, and vertical, OnBearing Vertical (OBV), directions. OSVM is measured in the tangential, On-Shaft Tangential (OST), and radial, On-Shaft Radial (OSR), directions. OBVM shows well separated and almost uncoupled horizontal and vertical responses. On the other hand, both onshaft sensors are affected by critical speeds equally. Moreover, OSVM shows high influence of gravity for OST and OSR as well as centrifugal acceleration for OSR.

Figure 7 shows the waterfalls of OBVM where the unbalance response clearly appears at $1 \mathrm{X}$ with peaks occurring as the speed crosses the critical values. Considerable $2 \mathrm{X}$ and $3 \mathrm{X}$ orders are also present. Figure 8 represents the waterfalls of OSVM where a nearly constant $1 \mathrm{X}$ order due to gravity can be detected. The resonances due to unbalance appear at the $2 \mathrm{X}$ harmonic. The DC components are excluded from the waterfalls. Both sensors are influenced when the speed passes the critical values.

Resampling the signal at constant angular increments [15] enabled extending the analysis to the orders domain which facilitates the extraction of the orders of interest for further examination.

Figure 9 shows $1 \mathrm{X}$ order for OBVM. Both horizontal and vertical responses are typical ones. Figure 10 shows $1 \mathrm{X}$ and $2 \mathrm{X}$ orders for OSVM. The $1 \mathrm{X}$ order is mainly due to gravity. The $2 \mathrm{X}$ order reflects the vibration increase across critical speeds. Their amplitudes are identical with some fluctuations at the critical speeds. Their phases are identical with $90^{\circ}$ shift. The sudden phase change corresponding to each critical speed appears in the phases of both sensors.

\section{Discussion}

The FE model described in Section 2.2 and the analytical model described in Section 3 are validated against experimental results.

Upon solving the equation of motion (Equation 10), the unbalance response is obtained as shown in

Figure 11. Compared against the experimental one in Figure 9, the critical speeds (characterized by amplitude amplifications and phase shifts) occurred at almost the same speeds noticed in the experimental run up. The sharp peaks and sudden phase transitions noticed in

Figure 11 may indicate slightly lower damping, however, this is expected to give better decoupling between the horizontal and vertical responses. The FE model simulated the unbalance amplitude and phase to an acceptable extent.

To validate the analytical model, Equation (11) is applied to the time waveforms of the unbalance response. Figure 12 shows the time waveforms of the simulated OSVM, Figure 13 shows the waterfalls (without DC components) and Figure 14 shows the $1 \mathrm{X}$ and $2 \mathrm{X}$ orders.

In both the experimental (Figure $6 \mathrm{c}$ and d) and simulated (Figure 12) time waveforms, the centrifugal acceleration is clearly affecting the radial sensors output and gravity is present in all sensors. Inspecting the waterfalls (Figure 13), gravity is again localized at the $1 \mathrm{X}$ order and the unbalance response at the $2 \mathrm{X}$ order. These two observations are the same as in Figure 8 . The only difference is the absence of the ridges at the $3 \mathrm{X}$ order. This may be referred to the fact that only unbalance is included in the FE model with almost no nonlinearities. 


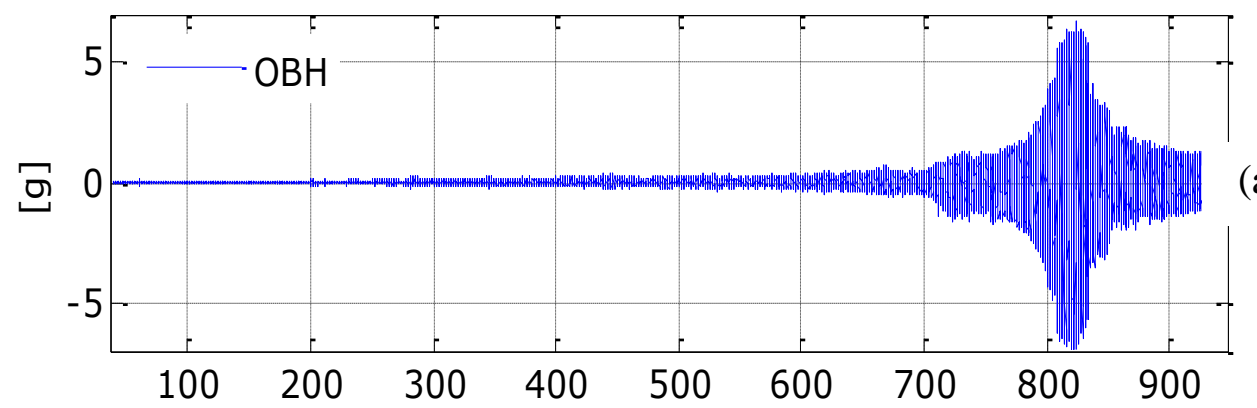

(a)

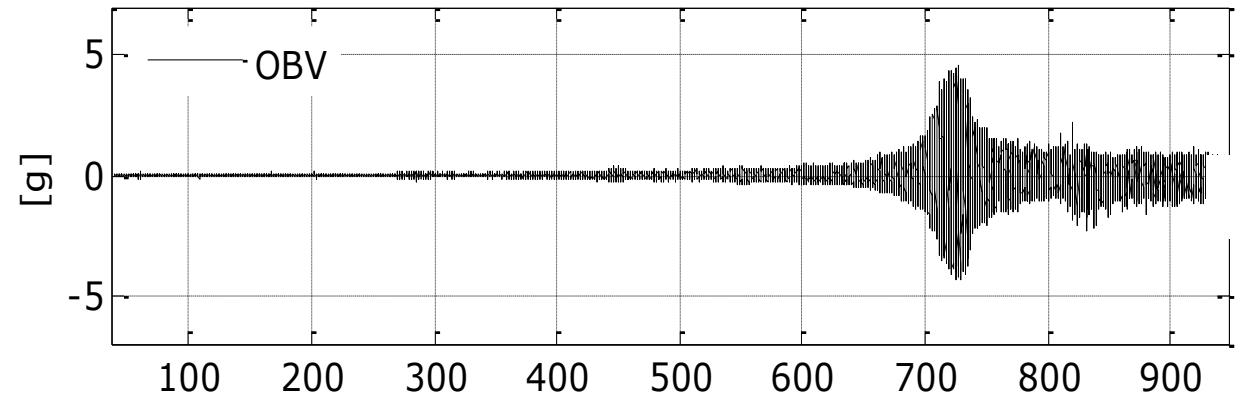

(b)

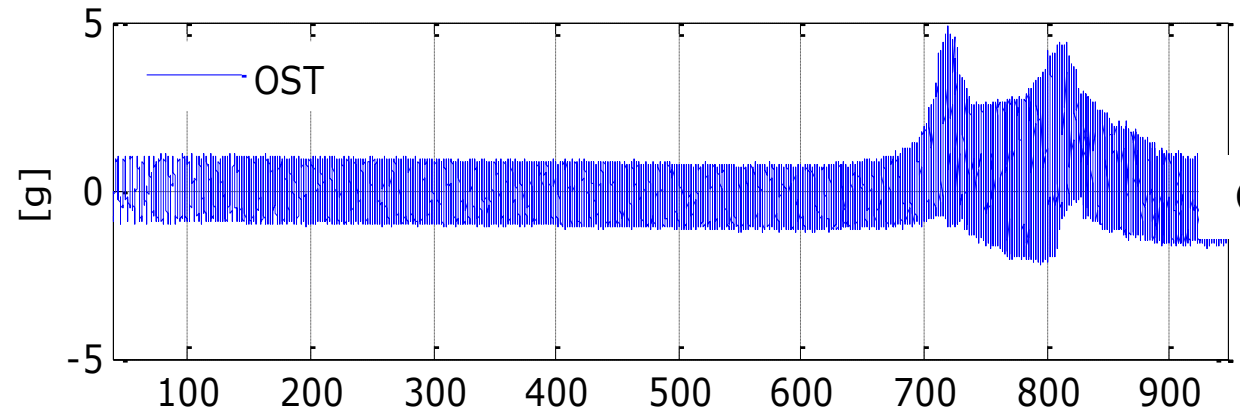

(c)

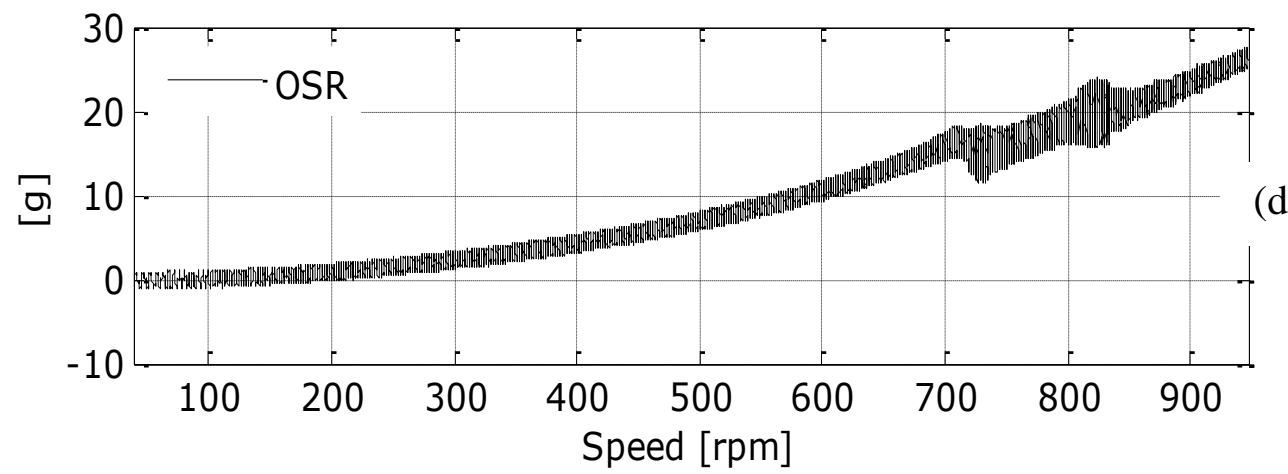

Figure 6: (a-b) Time waveforms of experimental OBVM (horizontal and vertical), and (c-d) OSVM (tangential and radial). 


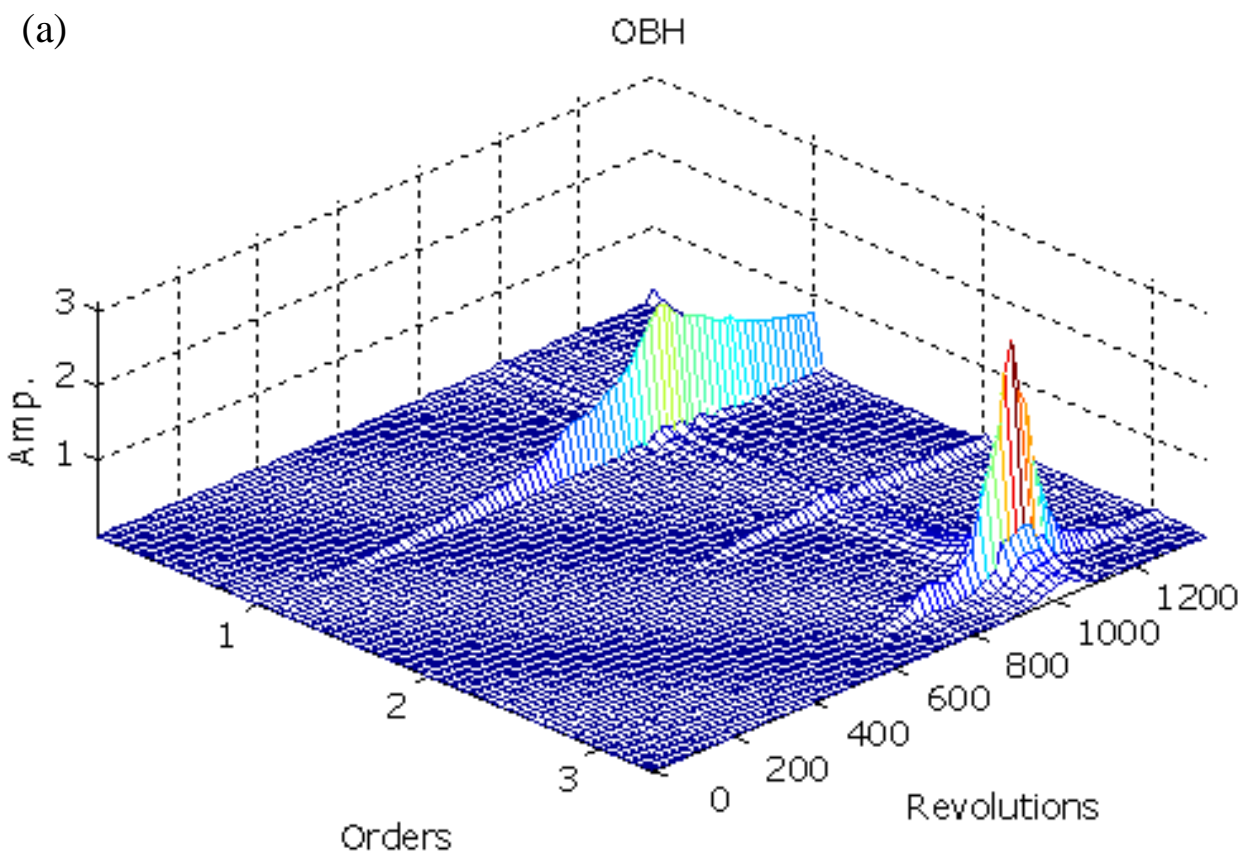

(b)

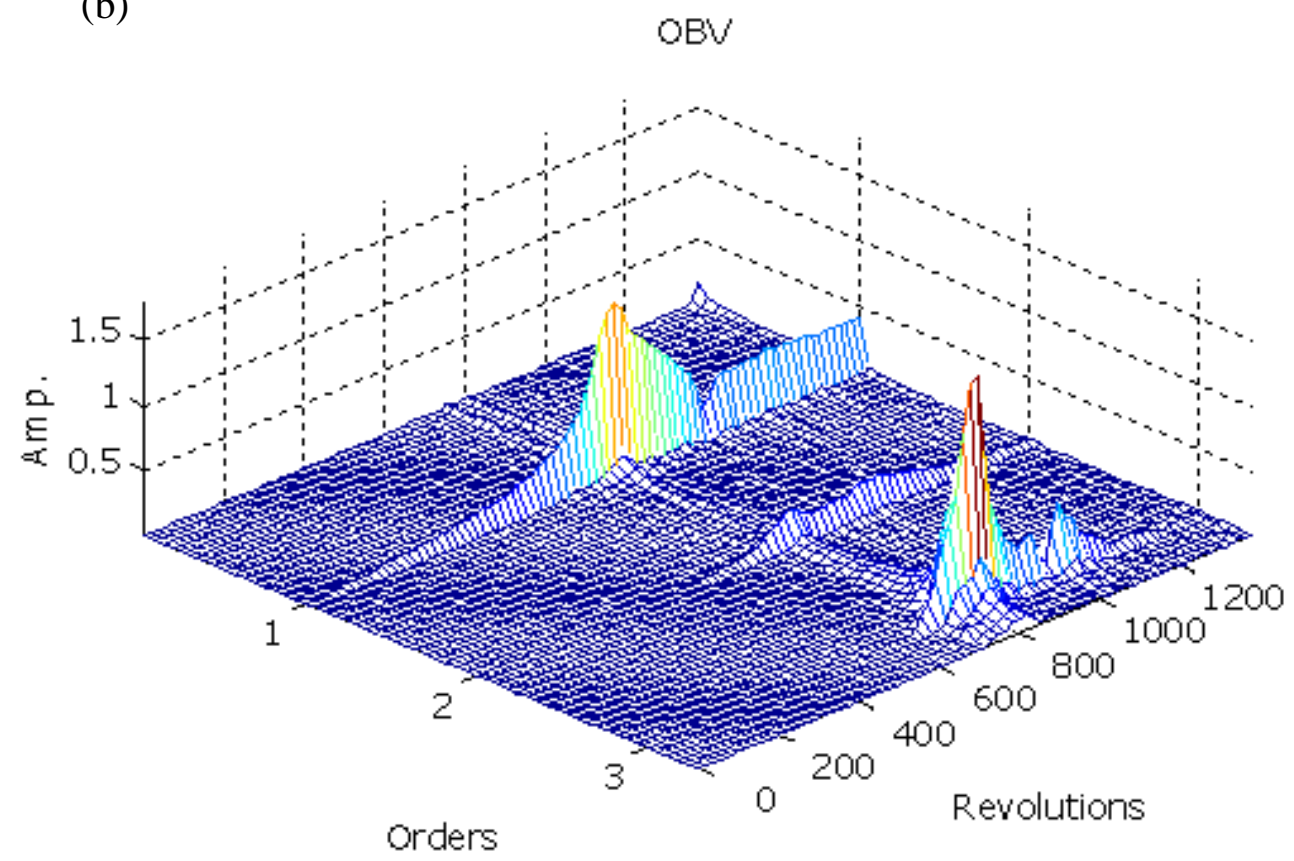

Figure 7: Waterfalls of experimental OBVM: (a) Horizontal, and (b) Vertical. 


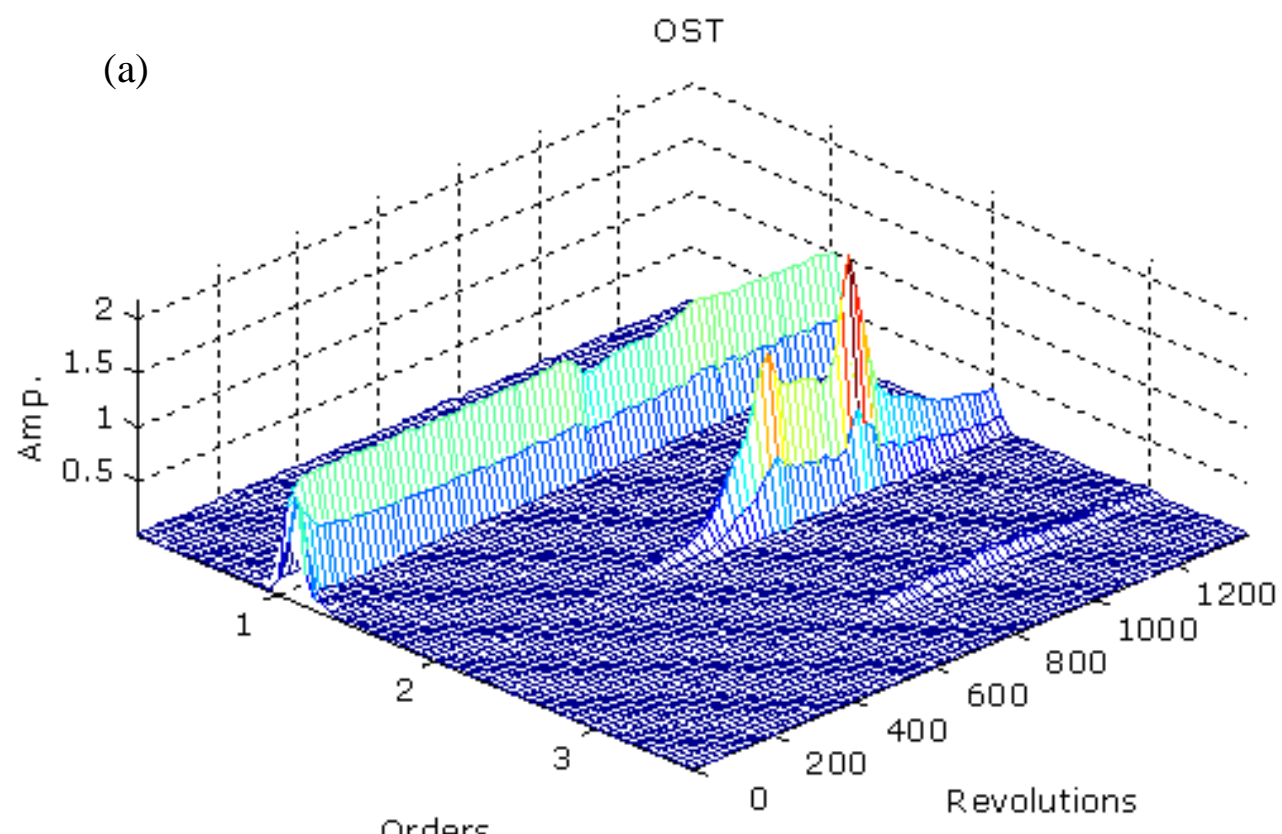

Orders

(b)

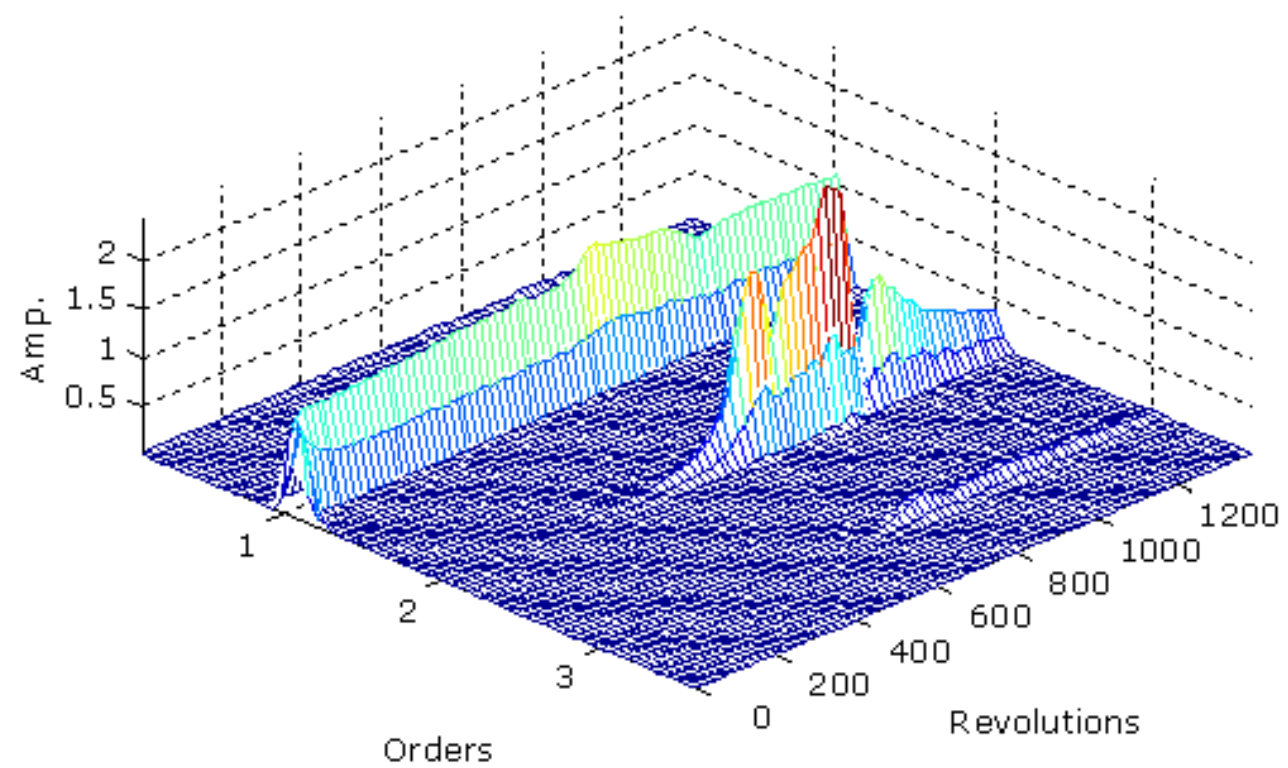

Figure 8: Waterfalls of experimental OSVM: (a) Tangential, and (b) Radial. 

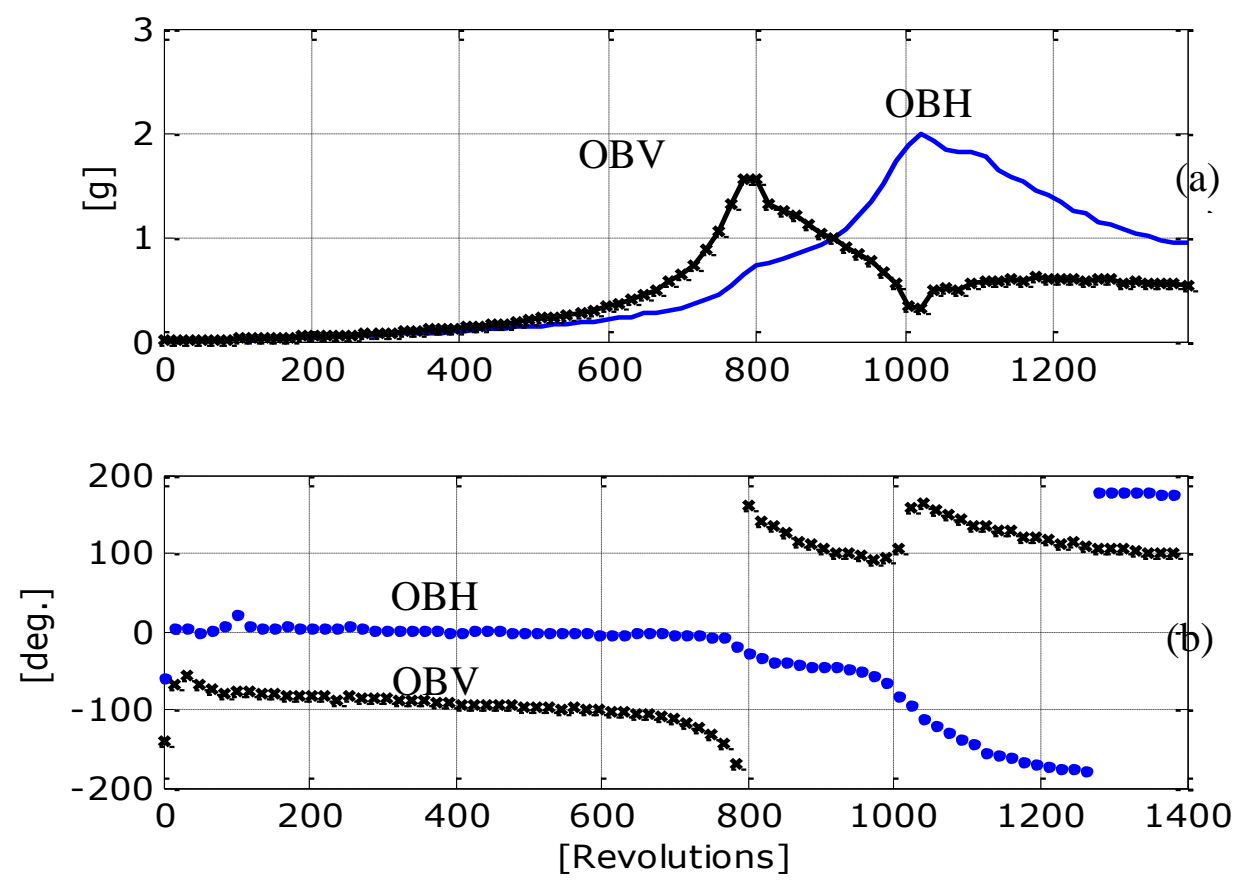

Figure 9: 1X order of experimental OBVM: (a) Amplitudes, and (b) Phases.

The 1X orders amplitudes and phases in Figure $14(\mathrm{a}-\mathrm{b})$ show good matching to the experimental ones in Figure $10(\mathrm{a}-\mathrm{b})$. The amplitudes and phases of the 1X order are nearly constant along the whole speed range. This indices that the unbalance response has absolutely nothing to do with the $1 \mathrm{X}$ order.

The simulated $2 \mathrm{X}$ orders amplitudes, Figure 14(c), show typical responses in the radial and tangential sensors. This contrasts to the experimental responses in Figure $10 \mathrm{c}$. This may be referred to additional effects from the $3 \mathrm{X}$ order noticed in Figure 7 which contributes to the resultant $2 X$ order when measured using OSVM. The simulated $2 X$ phases, Figure 14(d), show good matching with the experimental ones in Figure 10(d). The scattered readings at very low speeds may be due to resampling errors when moving to the orders domain. These erroneous points me be enhanced using higher sampling rates.

These comparisons between experimental and simulated OSVM recommend the derived analytical model to represent the OSVM process. Moreover, the following guidelines are expected to govern the OSVM process:

1) As the sensor rotates, modulation takes place where each order of OBVM splits into two parts at $+/-$ the rotational speed. For example, the nominal $1 \mathrm{X}$ order contributes to the modulated $\mathrm{DC}$ and $2 \mathrm{X}$ orders, the nominal $2 \mathrm{X}$ order contributes to the modulated $1 \mathrm{X}$ and $3 \mathrm{X}$ orders and so on.

2) The DC component of OSVM is influenced by changes in both amplitude and phase of the $1 \mathrm{X}$ order in OBVM.

3) Although OBVM shows uncoupled horizontal and vertical responses, OSVM provides coupled response. Each order in OSVM appears in both sensors OST and OSR with equal amplitudes and phases separated by $90^{\circ}$. 


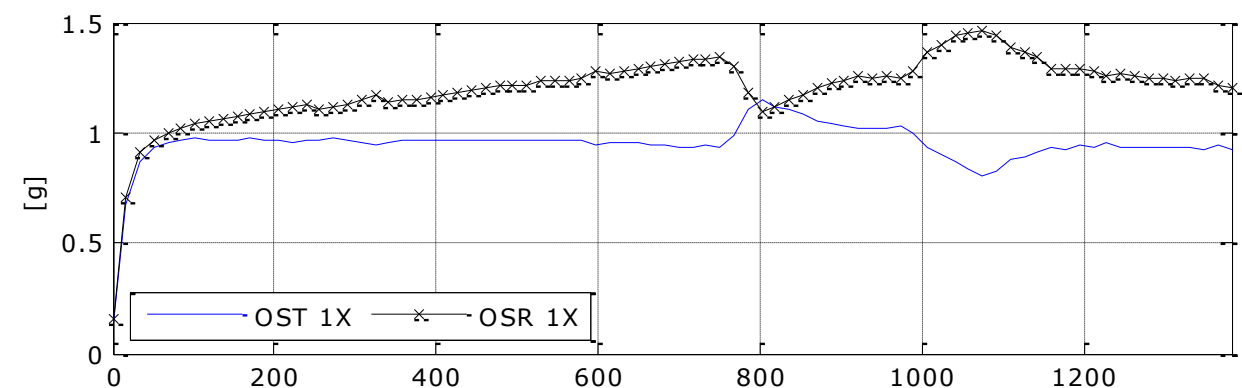

(a)

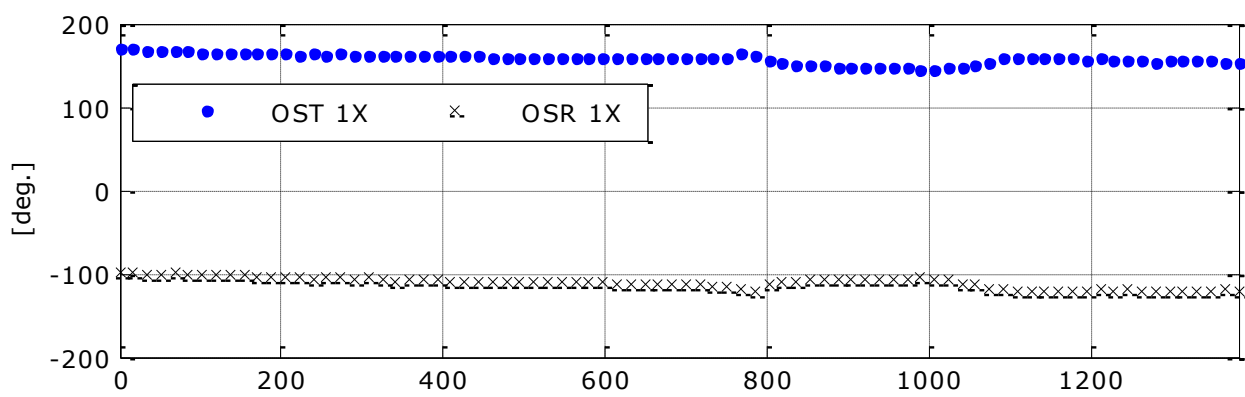

(b)

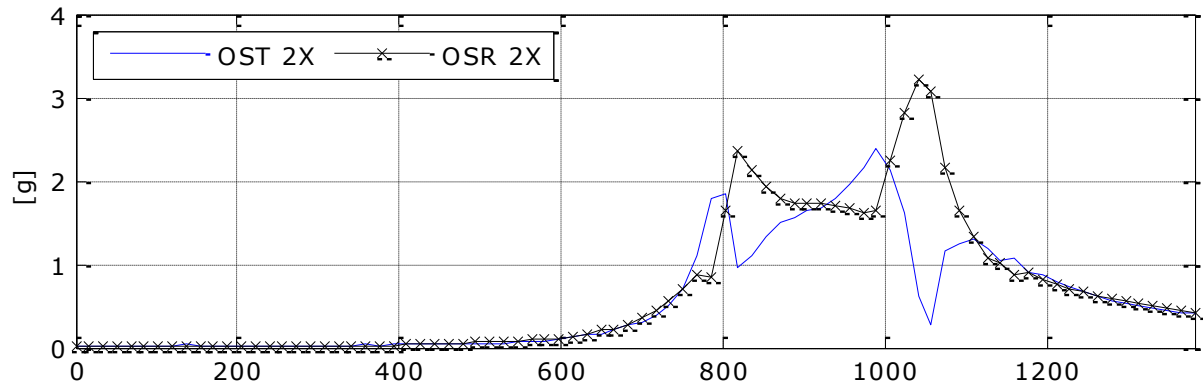

(c)

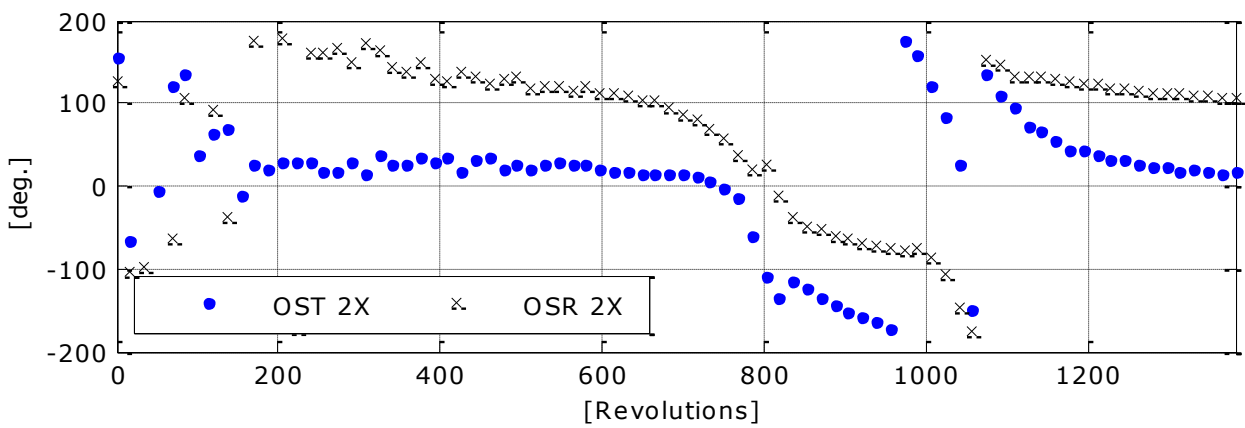

(d)

Figure 10: 1X and 2X orders of experimental OSVM: (a) 1X amplitudes, (b) 1X phases, (c) $2 \mathrm{X}$ amplitudes and (d) $2 \mathrm{X}$ phases. 


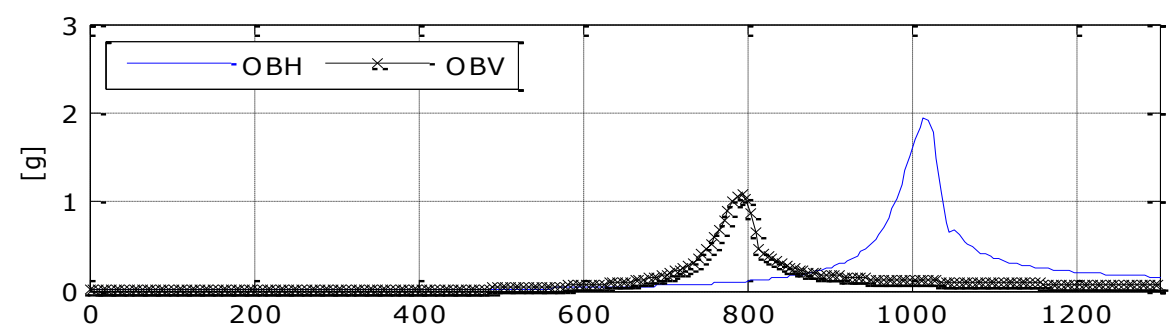

(a)

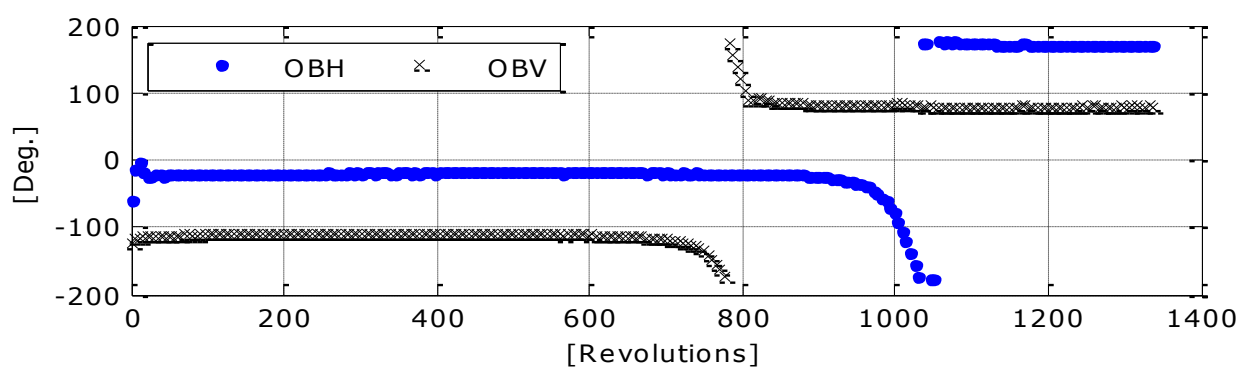

(b)

Figure 11: 1X order of simulated OBVM: (a) Amplitude (horizontal and vertical), and (b) phase (horizontal and vertical).

\section{Conclusions and Future Work}

In this work, experimental and FE models for a simple rotating machine are proposed. The comparisons between unbalance responses from $\mathrm{FE}$ and experimental run-ups show good matching. This suggests the FE model to be a good representation for the experimental test rig.

A simple analytical model is suggested to describe the OSVM process. The model is validated through experimental and FE results. The successful validation of the proposed model adds to the understanding of OSVM. The model also establishes some guidelines to help understanding how the OSVM process works.

We are currently working on implementing OSVM to diagnose rotating machine faults. The clear vibration signal and low sensitivity to noise from surrounding machines are expected to result in robust fault features.

Another suggestion for future work, in the case of multiple rotors, the number of on-shaft sensors may be optimised to acquire vibrations of adjacent rotors with the minimum number of sensors. 


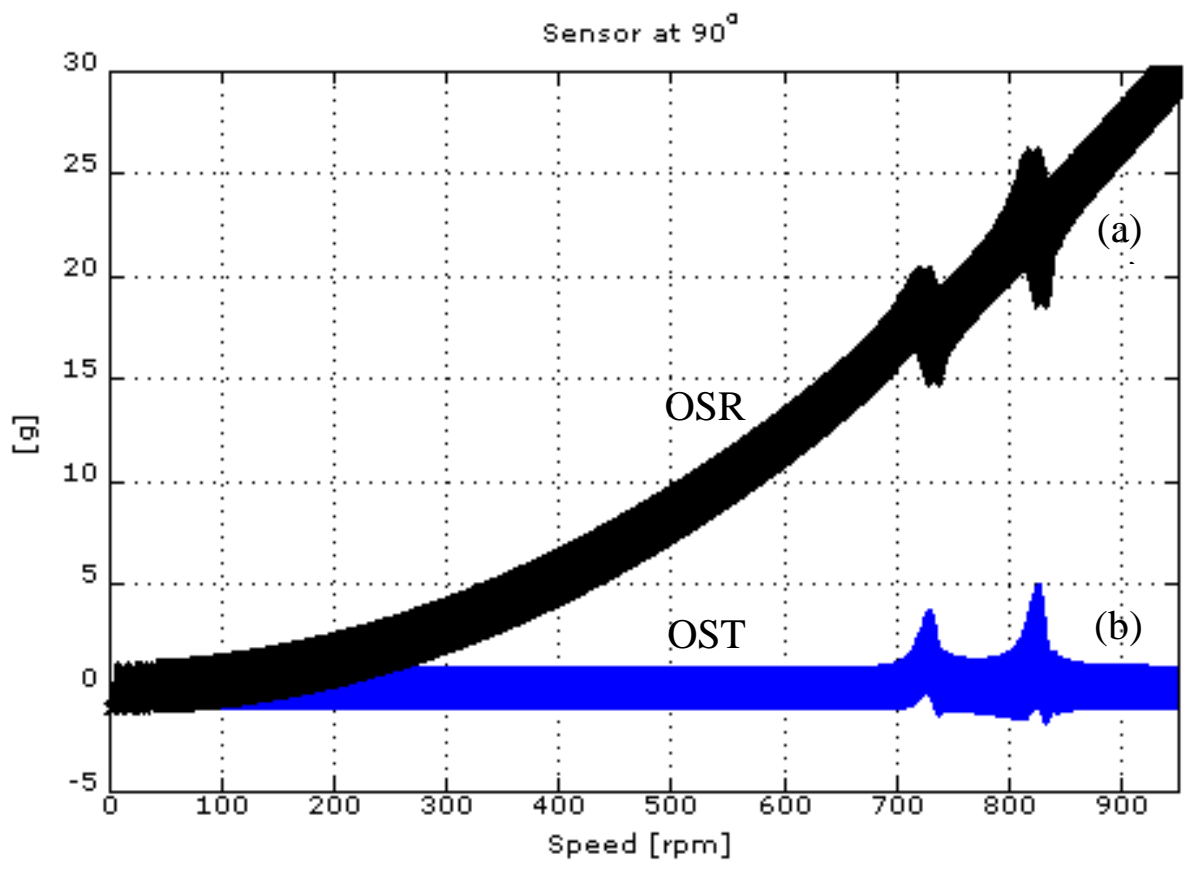

Figure 12: Time waveforms of simulated OSVM: (a) Radial and (b) Tangential.

(a)

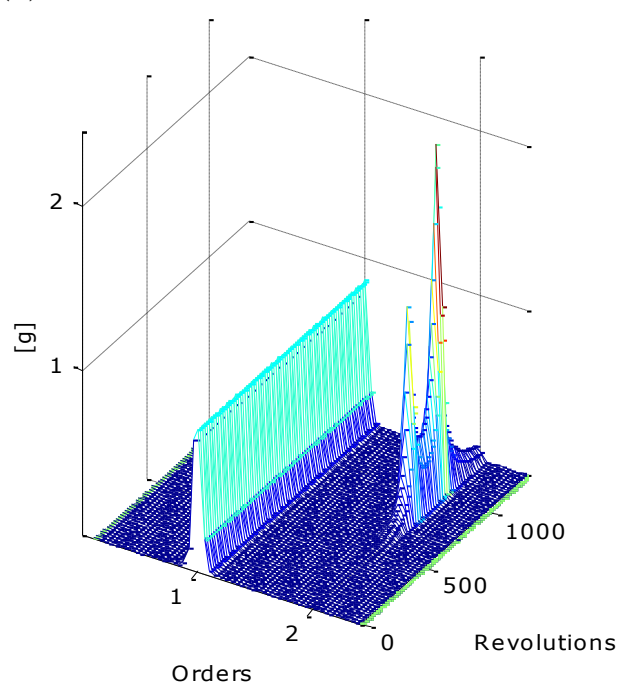

(b)

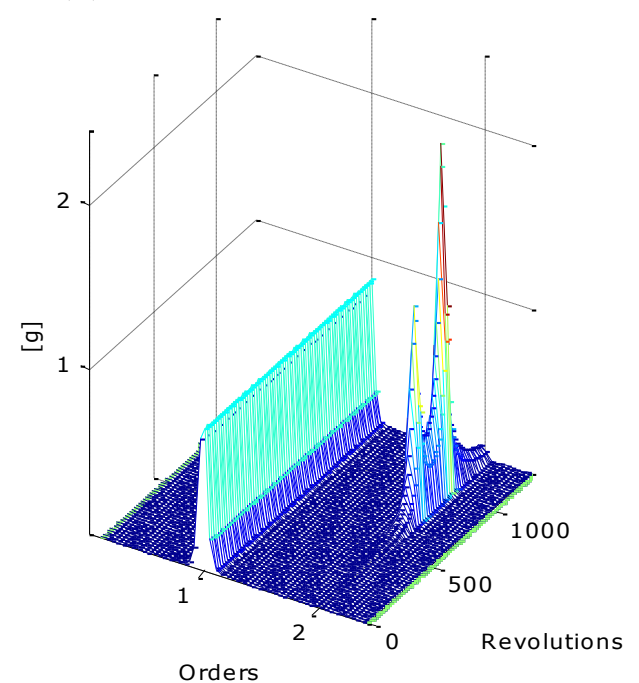

Figure 13: Waterfalls of simulated OBVM: (a) Tangential, and (b) Radial. 


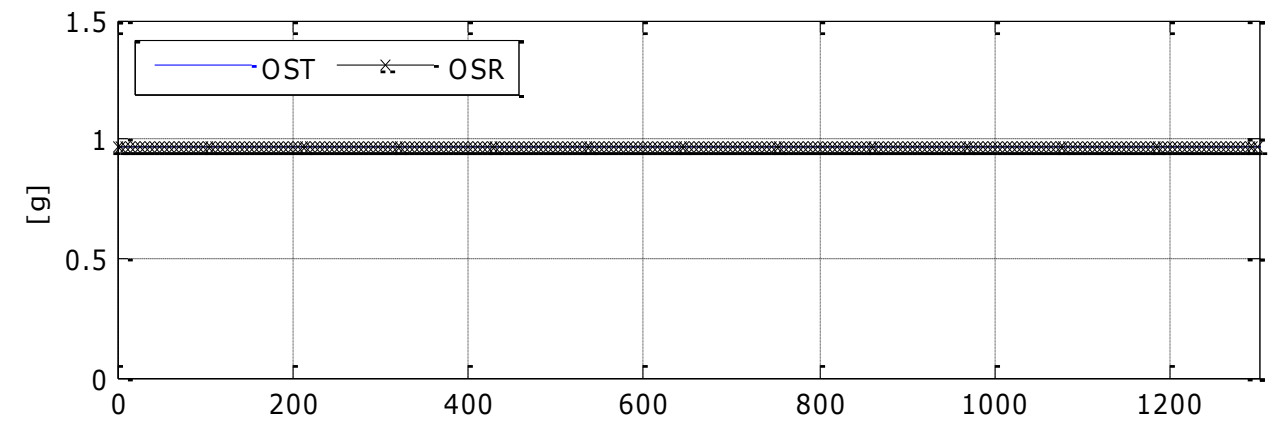

(a)

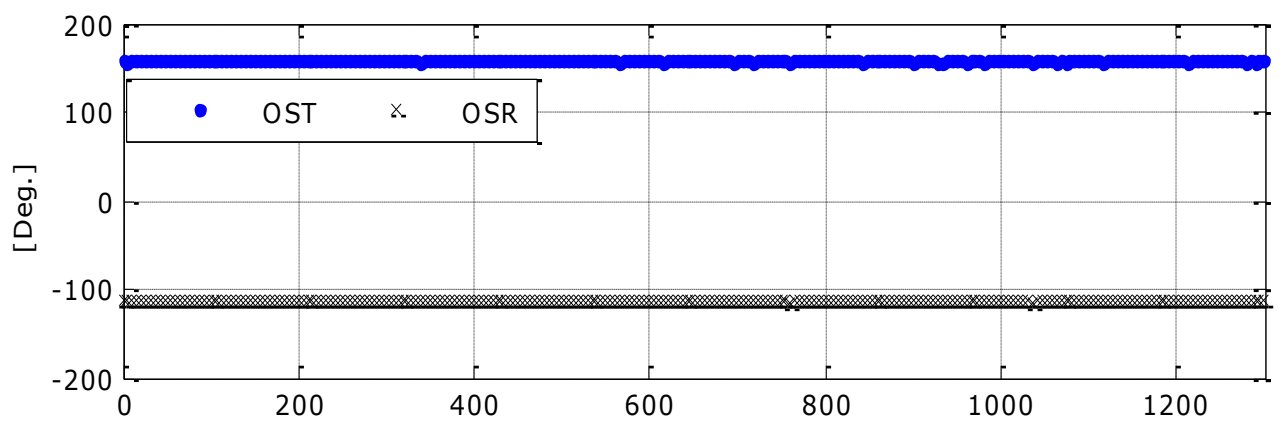

(b)

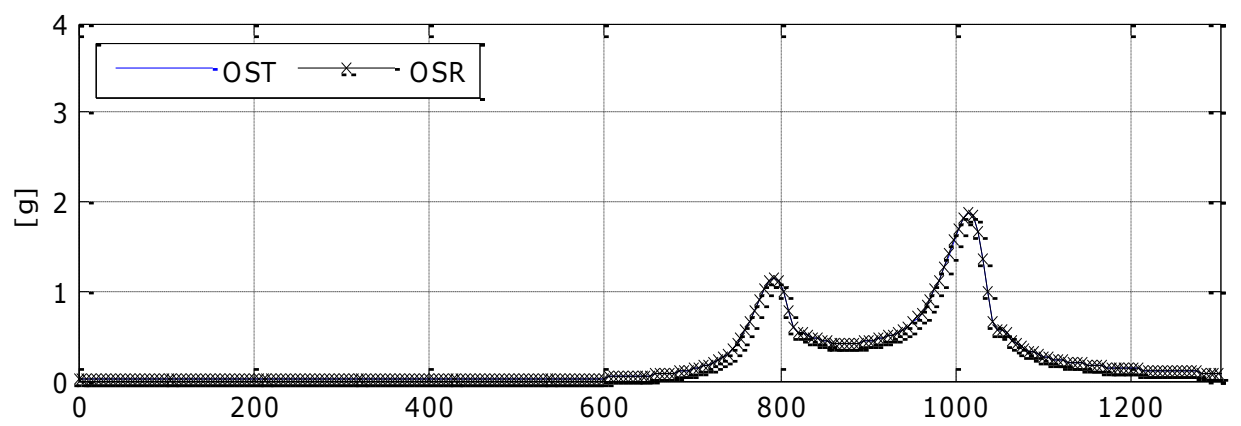

(c)

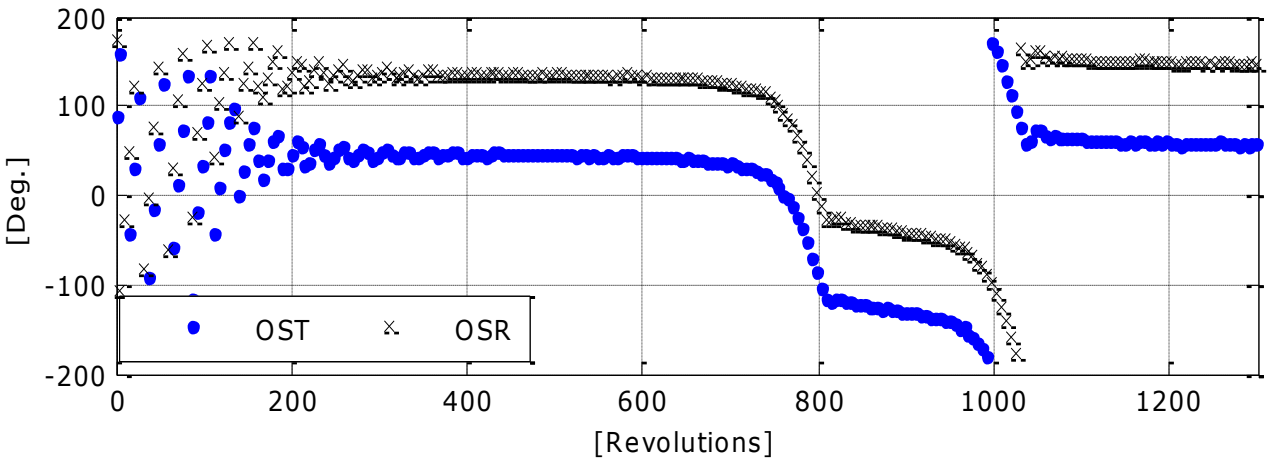

(d)

Figure 14: 1X and 2X orders of simulated OSVM: (a) $1 \mathrm{X}$ amplitude, (b) $1 \mathrm{X}$ phase, (c) $2 \mathrm{X}$ amplitude and (d) $2 \mathrm{X}$ phase. 


\section{References}

[1] J. S. Mitchell, An introduction to machinery analysis and monitoring Tulsa, Okla.: PennWell Pub. Co, 1981.

[2] Q. Liu and H. Wang, "A case study on multisensor data fusion for imbalance diagnosis of rotating machinery," Ai Edam-Artificial Intelligence For Engineering Design Analysis and Manufacturing, vol. 15, pp. 203-210, JUN 2001.

[3] W. Lees and K. A. Haines, "Torsional vibrations of a boiler feed pump," Journal of Mechanical Design, vol. 100, pp. 637-643, 1978.

[4] L. Naldi, M. Golebiowski, and V. Rossi, "Newapproachtotorsionalvibrationmonitoring", Proceeding of The Fortieth Turbomachinery Symposium, Houston, Texas, 2011.

[5] J. Urban, R. Christenson, and P. Mayzus. "Tracking the silent killer, torsional vibration, with high tech tools". Compressor TechTwo. 4, 2010.

[6] M. J. Z. Zhipeng Fenga, "Fault diagnosis of planetary gearboxes via torsional vibration signal analysis", Mechanical Systems and Signal Processing, In press.

[7] L. Arebi, F. Gu, N. Hu, and A. Ball, "Misalignment Detection Using a Wireless Sensor Mounted on a Rotating Shaft",Proceeding of the International congress on condition monitoring and diagnostic engineering management, 2011.

[8] L. Baghli, J. F. Pautex, and S. Mezani, "Wireless Instantaneous Torque Measurement, Application to Induction Motors",Proceeding of XIX International Conference on Electrical Machines - ICEM 2010, Rome, 2010.

[9] H. Thompson, "Wireless Sensor Research at the Rolls-Royce Control and Systems University Technology Center", $20091^{\text {st }}$ International Conference on Wireless Communication, Vehicular Technology, Information Theory and Aerospace \& Electronic Systems Technology, Vols 1 and 2, pp. 517-522, 2009.

[10] P. Sloetjes and A. de Boer, "Vibration reduction and power generation with piezoceramic sheets mounted to a flexible shaft", Journal of Intelligent Material Systems and Structures, vol. 19, pp. 25-34, JAN 2008.

[11] M. E. Elnady, J. K. Sinha, and S. O. Oyadiji, "On-Shaft Wireless Vibration Measurement for Condition Monitoring of Rotating Machines",Proceeding of the $10^{\text {th }}$ Biennial International Conference on Vibration Problems, PRAGUE, Czech Republic, 2011.

[12] M. E. Elnady, J. K. Sinha, and S. O. Oyadiji, "Identification of critical speeds of rotating machines using on-shaft wireless vibration measurement", $25^{\text {th }}$ International Congress on Condition Monitoring and Diagnostic Engineering Management (COMADEM12), Journal of Physics: Conference Series, Vol. 364, Issue 1, 2012.

[13] J.-J. Sinou, C. Villa, and F. Thouverez, "Experimental and Numerical Investigations of a Flexible Rotor on Flexible Bearing Supports," International Journal of Rotating Machinery, vol. 2005, pp. 179-189, 2005.

[14] M. I. Friswell, J. E. T. Penny, and S. D. Garvey, "Dynamics of Rotating Machines", Cambridge University Press, 2010.

[15] C. Groover, M. Trethewey, K. Maynard, and M. Lebold, "Removal of order domain content in rotating equipment signals by double resampling", Mechanical Systems and Signal Processing, vol. 19, pp. 483-500, MAY 2005. 